5. Für die beiden Förderpreise sind Selbstbewerbungen möglich; selbstverständlich sind Vorschläge von Dritten willkommen. Verlage sind nicht vorschlagsberechtigt.

6. Frauen sind besonders aufgefordert, sich zu bewerben; dies gilt auch für die Benennung von Kandidatinnen durch Dritte.

7. Die Einsendefrist für im Jahre 2005 veröffentlichte Publikationen ist der 31. März 2006.

Vorschläge und Benennungen für die Wissenschaftspreise bitten wir Sie, zusammen mit drei Exemplaren der Veröffentlichung und einer Kopie der Promotionsurkunde an die Geschäftsstelle der DVPW zu senden. Vorstand und Beirat werden die Vorschläge durch eine aus drei Kolleginnen und Kollegen bestehende Jury begutachten lassen.

Deutsche Vereinigung für Politische Wissenschaft, c/o Universität Osnabrück, FB 1 Sozialwissenschaften, D-49069 Osnabrück, www.dvpw.de

\title{
Wissenschaftspreis der Deutschen Vereinigung für Politische Wissenschaft für eine Arbeit aus dem Bereich der Genderforschung
}

Die Deutsche Vereinigung für Politische Wissenschaft schreibt ab dem Jahr 2006 alle drei Jahre zu ihrem wissenschaftlichen Kongress einen mit 2.000,00 EURO dotierten Förderpreis für eine wissenschaftliche Arbeit zu einem bestimmten Schwerpunkt aus. Der Preis für das Jahr 2006 wird für eine Arbeit aus dem Bereich der Genderforschung vergeben.

Folgende Bedingungen gelten:

1. Es können Monographien aus dem Bereich der Genderforschung eingereicht werden.

2. In Frage kommende Publikationen sollen in den Jahren 2003, 2004 oder 2005 veröffentlicht worden sein.

3. Der Kreis der Bewerberinnen und Bewerber ist auf Personen beschränkt, die ihre Arbeit in Deutschland publiziert haben, oder auf Deutsche, die ihre Monographie im Ausland veröffentlicht haben.

4. Für den Förderpreis sind Selbstbewerbungen möglich; selbstverständlich sind Vorschläge von Dritten willkommen. Verlage sind nicht vorschlagsberechtigt.

5. Frauen sind besonders aufgefordert, sich zu bewerben; dies gilt auch für die Benennung von Kandidatinnen durch Dritte.

6. Die Einsendefrist für im Jahre 2003, 2004 oder 2005 veröffentlichte Publikationen ist der 31. Januar 2006.

Bitte senden Sie Ihre Bewerbungen oder Vorschläge für den Wissenschaftspreis zusammen mit drei Exemplaren der Veröffentlichung an die Geschäftsstelle der DVPW. Vorstand und Beirat werden die Vorschläge durch eine aus drei Kolleginnen und Kollegen bestehende Jury begutachten lassen.

Deutsche Vereinigung für Politische Wissenschaft, c/o Universität Osnabrück, FB 1 Sozialwissenschaften, D-49069 Osnabrück, www.dvpw.de 